\title{
ORIGEM E DISTRIBUIÇÃO DA ARTÉRIA CELÍACA DE AVESTRUZES (Struthio camelus)
}

\author{
Bruno Gomes Vasconcelos ${ }^{1}$, Frederico OZANam Carneiro e Silva ${ }^{2}$, Renata Lima de \\ Miranda $^{3}$, Cheston Cesar Honorato Pereira ${ }^{4}$, Amilton Cesar dos Santos ${ }^{1}$, Maria Angelica \\ MIGLINO $^{5}$
}

${ }^{1}$ Pós-graduando da Faculdade de Medicina Veterinária e Zootecnia da Universidade de São Paulo, São Paulo, SP, Brasil. brunog_vet@hotmail.com

${ }^{2}$ Professor Doutor da Universidade Federal de Uberlândia, Uberlândia, MG, Brasil

${ }^{3}$ Pós-graduanda da Universidade Federal de Uberlândia, Uberlândia, MG, Brasil

${ }^{4}$ Professor Mestre da Universidade de Rio Verde, Rio Verde, GO, Brasil

${ }^{5}$ Professora Doutora da Universidade de São Paulo, SP, Brasil.

A exploração de avestruzes visa à comercialização das carnes, penas, couro e gordura. Intimamente relacionado à produtividade está o sistema digestório e, dentre os importantes vasos responsáveis pela sua nutrição, está a artéria celíaca, cuja origem e distribuição foi aqui estudada. Em 30 exemplares, a artéria isquiática esquerda foi canulada para injeção de solução marcadora de vasos sanguíneos, em seguida, fixada em solução aquosa de formol 10\%, mediante aplicações intramuscular profunda, subcutânea e intracavitária. Concluímos que ela originouse da aorta descendente, sendo o seu primeiro ramo ventral e dirigindo-se para o antímero direito em seu trajeto enviou vasos para: esôfago, proventrículo, ventrículo, baço, fígado, vesícula biliar, pâncreas, duodeno, íleo, cecos esquerdo e direito.

PALAVRAS CHAVES: aves; irrigação; sistema digestório.

\section{ORIGIN AND DISTRIBUTION OF THE CELIAC ARTERY IN OSTRICH (Struthio camelus)}

\section{ABSTRACT}

The exploration of ostriches aims at trading meat, feathers, leather and grease. The digestive system is intimately related to productivity, and the celiac artery is amongst the major vessels responsible for its nutrition. The artery's origin and distribution was studied here. In 30 specimens, the left ischiatic artery was cannulated for the injection of a blood vessels marker solution and then fixed in formalin solution $10 \%$ via deep intramuscular, subcutaneous and intracavitary applications. We concluded that this artery originated from the descending aorta, its first branch is ventral and it headed for the right side sending branches to: esophagus, proventriculus, ventriculus, spleen, liver, gallbladder, pancreas, duodenum, ileum, left and right cecum.

KEYWORDS: birds; digestive system; irrigation. 


\section{INTRODUÇÃO}

As ratitas são aves corredoras que apresentam características anatômicas e fisiológicas que as diferenciam das aves carinatas (aves que voam), ou seja, são incapazes de voar, não possuem musculatura no peito para vôo e nem quilha sobre o osso esterno (SICK, 1997). Dentre elas, as espécies mais exploradas comercialmente são o avestruz, o emu e a ema (GIANNONI, 1998).

O avestruz é a ave corredora de maior tamanho que existe no mundo, com pernas largas, robustas e pés com apenas dois dedos. Seu nome foi originado da junção de duas palavras: ave + estruz, derivado do latim Struthio camelus, que significa pássaro camelo (CARBÓ, 2003).

$\mathrm{Na}$ atividade pecuária, a carne do avestruz, $\mathrm{o}$ principal produto gerado, apresenta coloração escura bem característica e vem ganhando popularidade em diversas partes do mundo. A pele é o segundo subproduto do avestruz que encontrou um mercado restrito, porém expansivo e, por último, encontramse as plumas, consideradas como um subproduto marginal, que são amplamente comercializadas no carnaval (CARBÓ, 2003).

O sistema digestório está intimamente relacionado com a produtividade dos avestruzes, pois é nele que ocorre o processamento dos alimentos. O reconhecimento de suas estruturas e fisiologia é fundamental para se estabelecer uma nutrição mais adequada, voltada às particularidades digestivas, visando à obtenção de uma ração de alta digestibilidade para que ocorra um melhor aproveitamento pelo animal, proporcionando melhor conversão alimentar e, consequentemente, aumentando sua produção (MIRANDA et al., 2009).

Sendo assim, o crescimento da estrutiocultura no Brasil e a escassez de estudos sobre a vascularização dos órgãos do sistema digestório nessa espécie nortearam a realização deste estudo, cujo objetivo é determinar a origem e distribuição da artéria celíaca em avestruzes.

\section{MATERIAL E MÉTODOS}

Utilizaram-se 30 exemplares de avestruzes (S. camelus) de criatórios do município de Uberlândia-MG, obtidos de mortes naturais com uma semana de idade.

Após a morte natural das aves, a artéria isquiática esquerda foi canulada para injeção de solução aquosa a $50 \%$ de látex Neoprené " 450 ” (Du pont do Brasil. Indústrias Químicas. São Paulo SP), corada com pigmento específico de cor vermelha (Globo S/A Tintas e Pigmentos). Em seguida, as aves foram fixadas em solução de formol a 10\%, mediante aplicações intramuscular profunda, subcutânea e intracavitária, sendo posteriormente, mantidas submersas na mesma solução, tendo como intervalo mínimo para dissecação o período de 48 horas.

Para dissecação da artéria celíaca, utilizaram-se os instrumentos cirúrgicos adequados, auxiliados, quando necessário, pelo campo visual de uma lupa monocular tipo Wild (10X).

Subsequentemente às dissecações, foram realizados esquemas de cada exemplar, em que se registraram a origem, o número e a distribuição da artéria citada. Ainda confeccionaramse fotografrafias (Figura 1) e um desenho esquemático (Figura 2) para melhor ilustração dos resultados.

Análises descritivas de porcentagem simples dos dados foram utilizadas para se verificar diferenças estatísticas entre eles.

A nomenclatura adotada para descrição dos resultados foi a Nomina Anatomica Avium (Baumel, 1993).

O trabalho foi submetido e aprovado pela Comissão de Ética na Utilização de Animais da Universidade Federal de Uberlândia, sob o número de protocolo 079/10.

\section{RESULTADOS}

A artéria celíaca originou-se da aorta descendente, sendo o seu primeiro ramo ventral e dirigiu-se para o antímero direito. Em seu trajeto ela enviou ramos para: esôfago, proventrículo, ventrículo, baço, fígado, vesícula biliar, pâncreas, duodeno, íleo e cecos esquerdo e direito.

Após a origem, emitiu as artérias proventricular dorsal e lienal, em todos os exemplares, sendo que a primeira irrigou o esôfago em $10 \%$ e o baço em $3,3 \%$. Em seguida, a artéria celíaca dividiu-se em ramos direito e esquerdo 
(Figura 1).

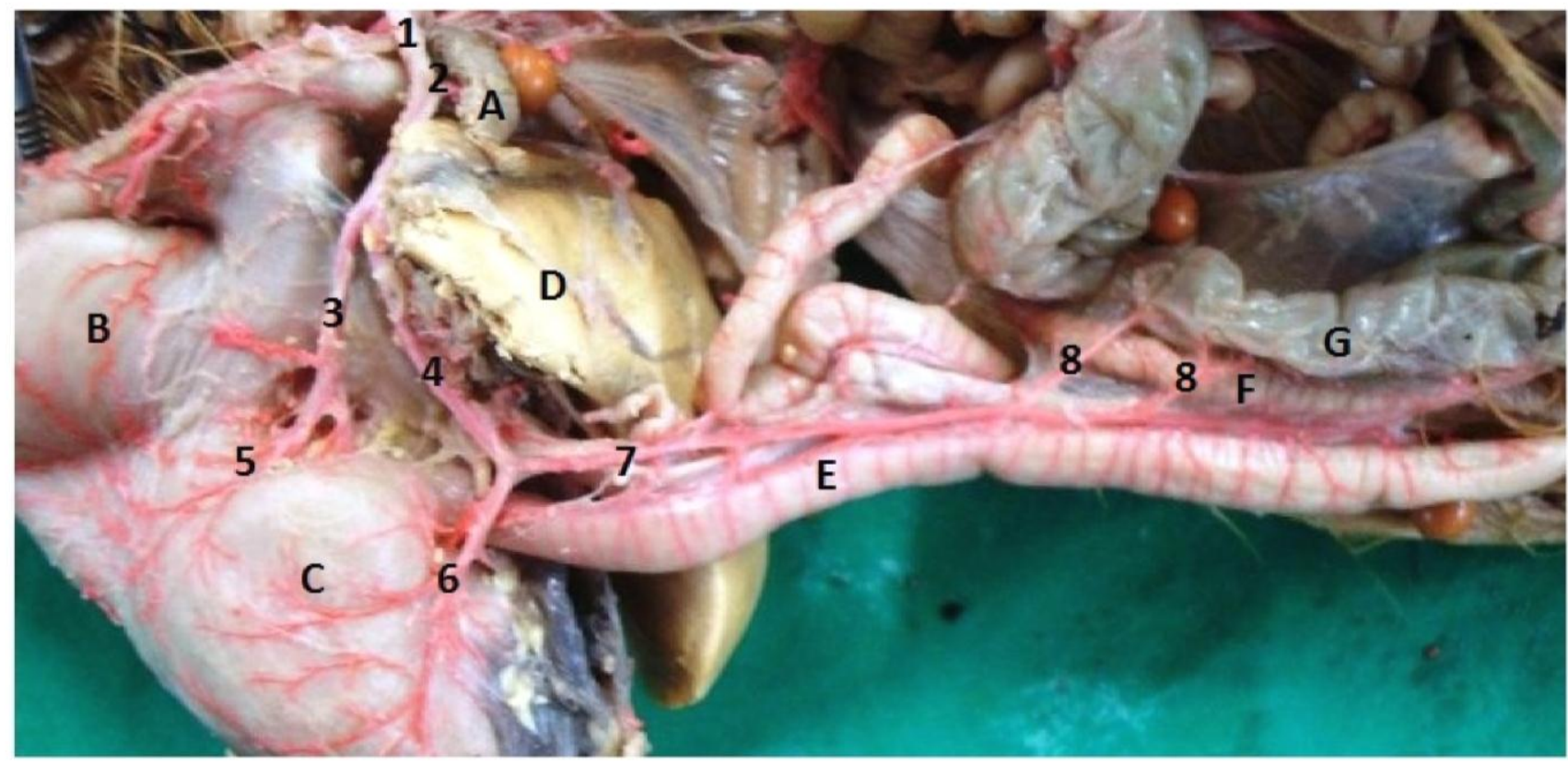

Figura 1. Fotomacrografia da cavidade celomática esquerda de avestruzes (S. camelus), sendo os órgãos: A baço; B - proventrículo; C - ventrículo; D - fígado; E - duodeno; F - íleo e G - ceco esquerdo; e a ramificação da A. celíaca (1) em: A. lienal (2), ramos esquerdo (3) e direito (4), A. gástrica esquerda (5), A. gástrica dorsal (6), A. pancreaticoduodenal (7) e Aa. ileocecais (8).

O ramo esquerdo enviou as artérias proventricular ventral (90\%), gástricas esquerda e ventral (100\%) (Figura 1) e ramos esofágicos $(33,3 \%)$. O fígado recebeu suprimento arterial diretamente do ramo esquerdo através da artéria hepática esquerda $(13,3 \%)$ e indiretamente do ramo esofágico $(3,3 \%)$ emitido pelo ramo esquerdo.

O ramo direito da artéria celíaca ramificouse nas artérias hepática direita, da vesícula biliar e gástrica direita e dorsal (Figura 1), em todas as aves. Próximo da transição gastroduodenal, o ramo direito emitiu ramos esofágico $(3,3 \%)$, duodenal $(13,3 \%)$ e pancreático (46,6\%); e continou como artéria pancreaticoduodenal (Figura 1), que seguiu seu trajeto entre a alça duodenal, vascularizou o duodeno e o pâncreas e terminou enviando as artérias ileocecais (Figura 1) que irrigaram o íleo $(86,6 \%)$ e ceco esquerdo (100\%) e direito $(96,6 \%)$.

Com o intuito de facilitar o entendimento da distribuição da artéria celíaca foi realizado um desenho esquemático (Figura 2). 


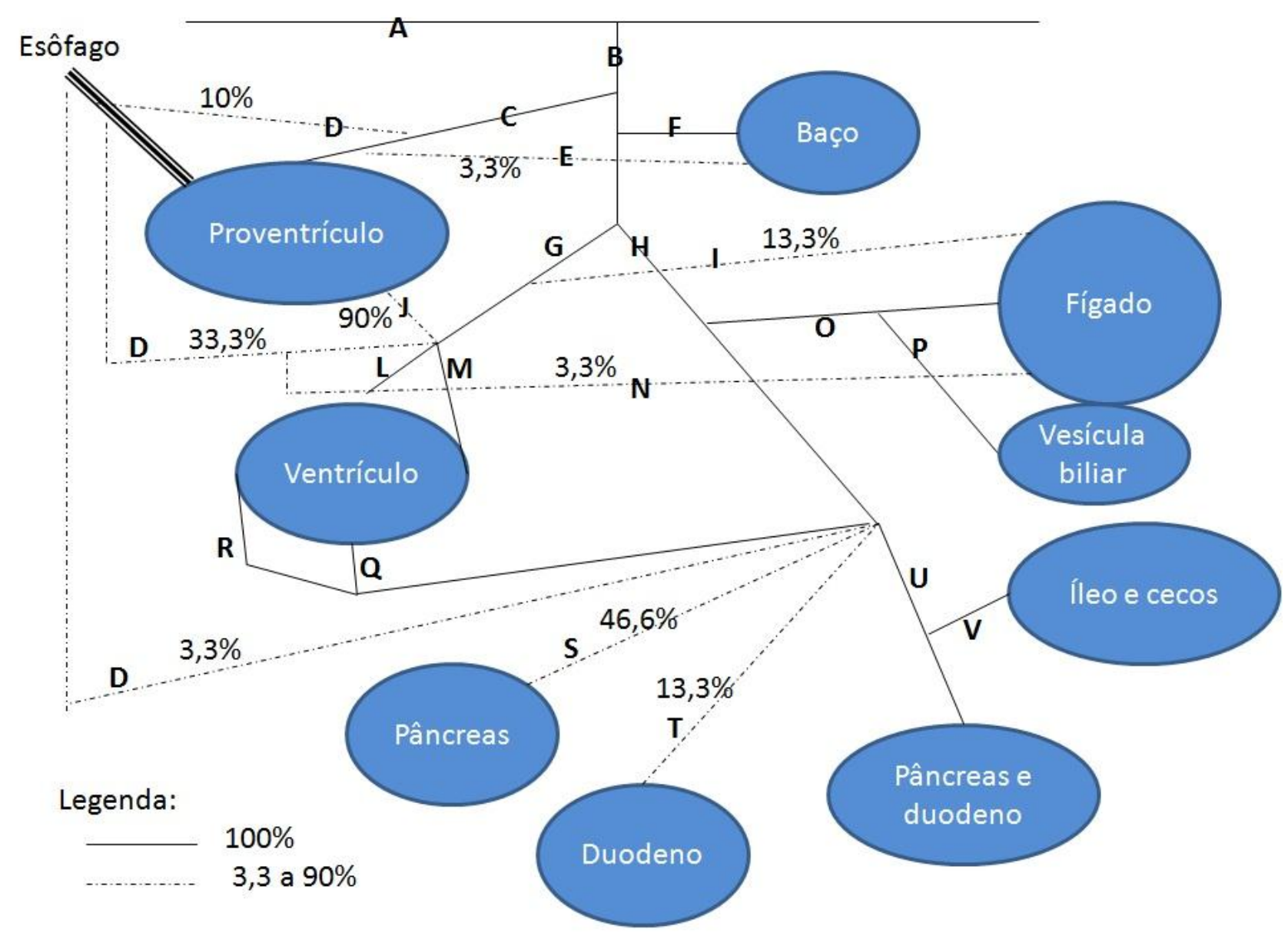

Figura 2. Esquema da origem e distribuição da artéria celíaca em avestruzes, sendo: A - aorta descendente; B - A. Celíaca; C - A. proventricular dorsal; D - ramo esofágico; E - ramo lienal; F - A. Lienal; G - ramo esquerdo; H - ramo direito; I - A. hepática esquerda; J - A. proventricular ventral; L - A. gástrica ventral; M - A. gástrica esquerda; $\mathrm{N}$ - ramo hepático; $\mathrm{O}$ - A. hepática direita; $\mathrm{P}-\mathrm{A}$. vesícula biliar; $\mathrm{Q}$ - A. gástrica dorsal; $\mathrm{R}$ - A. gástrica direita; $\mathrm{S}$ - ramo pancreático; $\mathrm{T}$ - ramo duodenal; $\mathrm{U}-\mathrm{A}$. pancreáticoduodenal e $\mathrm{V}-$ A. ileocecal.

As artérias lienais variaram de um a dois vasos, sendo um correspondente a $80 \%$ e dois a $20 \%$. O número de ramos para o proventrículo oriundo da artéria proventricular dorsal, variaram de dois a 18 vasos, sendo dois, cinco, 13, 16 e 18 equivalentes a $3,3 \%$, quatro, sete e 17 a $6,6 \%$, seis e oito a $10 \%$, dez e 14 a $13,3 \%$ e 12 a $16,6 \%$; a artéria proventricular dorsal enviou colaterais ao esôfago (10\%) e ao baço $(3,3 \%)$, emitindo um e dois ramos, respectivamente. Além disso, o proventrículo foi irrigado pela artéria proventricular ventral emitida pelo ramo esquerdo da artéria celíaca em $90 \%$ das aves, variando de um a nove, sendo sete e nove correspondentes a $3,7 \%$, três e oito a $7,4 \%$, um a $11,1 \%$, cinco e seis a $14,8 \%$ e dois e quatro a $18,5 \%$.

Posteriormente, o ramo esquerdo dividiu-se em artérias gástrica esquerda e ventral (100\%) e ramos esofágicos $(33,3 \%)$. A artéria gástrica esquerda enviou de dois a cinco colaterais, sendo que $13,3 \%$ aprensentaram cinco; $16,6 \%$, quatro; $26,6 \%$, três e $43,3 \%$, dois; e a gástrica ventral de três a 15 , sendo que 3,3\% presentaram três, quatro, 14 e 15; $6,6 \%$, seis e dez; $10 \%$, nove e $12 ; 13,3 \%$, cinco; e $20 \%$, sete e oito; já o ramo esofágico apresentou-se em número de um.

O fígado recebeu suprimento arterial diretamente do ramo esquerdo através da árteria hepática esquerda $(13,3 \%)$, obtendo de um a dois 
vasos, sendo que $50 \%$ apresentaram um e dois; e, indiretamente, através de um ramo esofágico $(3,3 \%)$ emitido pelo ramo esquerdo.

O ramo direito enviou, em todas as aves, a artéria hepática direita que variou de um a dois colaterais, $90 \%$ e $10 \%$, respectivamente. Anteriormente à ramificação da artéria hepática direita no parênquima hepático, ela emitiu a artéria da vesícula biliar que recebeu um ramo em todos os casos.

Em seguida, o ramo direito distribuiu vasos no ventrículo através das artérias gástrica direita e dorsal. A primeira variou de dois a seis colaterais, na seguinte proporção: quatro e seis, $10 \%$; cinco, $20 \%$; três, 26,6\%; e dois, 43,3\%. Já a artéria gástrica dorsal variou de dois a 14, sendo dois, quatro e 14 equivalentes a $3,3 \% ; 12$ a $6,6 \%$; cinco e nove a $10 \%$; sete e 10 a $13,3 \%$; seis a $16,6 \%$; e oito a $20 \%$. Em um espécime $(3,3 \%)$, o ramo direito terminou enviando um ramo esofágico.

Próximo da transição gastroduodenal, o ramo direito emitiu ramo esofágico $(3,3 \%)$ através de um colateral; ramo duodenal $(13,3 \%)$ apresentou de um a dois, sendo $50 \%$ com dois; e ramo pancreático (46,6\%), variando de um a cinco ramos, na proporção de $7,1 \%$ um e cinco, $14,2 \%$ dois, $28,5 \%$ quatro e $42,8 \%$ três.

O ramo direito da artéria celíaca continuou como artéria pancreaticoduodenal que irrigou o pâncreas e o duodeno e, para o primeiro, enviou de nove a 18 ramos, sendo verificado $3,3 \%$ com 11 ; 6,6\% com nove; $10 \%$ com 12, 14 e 17; 13,3\% com dez e 13; e 16,6\% com 15 e 19. Para o segundo enviou de 15 a 59 vasos, sendo 3, 33\% com 15, 18, $21,24,31,32,41$ e $59 ; 6,6 \%$ com 19, 20, 34 e 38; $10 \%$ com 25 e 27; 13,3\% com 26 e 28.

As artérias ileocecais originaram-se da artéria pancreaticoduodenal e apresentaram-se em número de uma $(90 \%)$ ou duas $(10 \%)$, e irrigaram o íleo $(86,6 \%)$, ceco esquerdo $(100 \%)$ e direito $(96,6 \%)$. No íleo, os vasos variaram de um a 10 , sendo $3,8 \%$ com $10,7,6 \%$ com seis e sete, $11,5 \%$ com quatro, $15,3 \%$ com um e cinco, $19,2 \%$ com dois e três; no ceco esquerdo, variaram de dois a 10 , sendo 3,3\% com dois, três, cinco e dez, $10 \%$ com quatro, $16,6 \%$ com oito, $20 \%$ com seis e $40 \%$ com sete; e no ceco direito variaram de dois a nove, sendo $3,4 \%$ com três e nove, $6,8 \%$ com quatro e sete,
$10,3 \%$ com dois, $20,6 \%$ com cinco e oito e $27,5 \%$ com seis.

\section{DISCUSSÃO}

Segundo SISSON \& GROSSMAN (1975), na galinha doméstica (Gallus gallus),

a artéria celíaca é um vaso impar da aorta, EDE (1965) descreveu sua origem no extremo anterior da cavidade peritonial, NICKEL et al. (1977) a consideraram como sendo o primeiro grande ramo ventral da aorta e BAUMEL (1993) referiu-se a ela como um vaso originário da aorta descendente. SCHWARZE \& SCHRODER (1972) e GETTY (1981) em G. gallus e SILVA et al. (1996) na linhagem Hubbard relataram que a artéria referida é oriunda da face ventral da aorta descendente, com o que concordamos e ainda acrescentamos que, no caso do avestruz, ela se dirige para o antímero direito.

O ramo esofágico, originário da artéria celíaca logo após a sua origem, foi citado por BHADURI et al. (1957), SCHWARZE \& SCHRODER (1972) e NICKEL et al. (1977), em $G$. gallus; DRUMMOND et al. (2000) em G. gallus domesticus e SILVA et al. (2001) na linhagem Avian Farms. MIRANDA et al. (2005) na Redbro Plumé, complementaram que foi encontrado em $80 \%$ do exemplares. Entretanto BAUMEL (1993) em $G$. gallus, citou que esses ramos foram emitidos pela artéria proventricular dorsal, com o que estamos de acordo, e esteve presente em 10\% das aves, porém encontramos ainda a irrigação através do ramo esquerdo da artéria celíaca em $33,3 \%$ e do direito em $3,3 \%$.

Os ramos para o proventrículo, referidos por BHADURI et al. (1957), SCHWARZE \& SCHRODER (1972), SISSON \& GROSSMAN (1975), GETTY (1981) e BAUMEL (1993) em $G$. gallus; DRUMMOND et al. (2000) no G. gallus domesticus e SILVA et al. (2001), na Avian Farms, foram encontrados em $100 \%$ das aves. MIRANDA et al. (2005) acrescenta que, na Redbro Plumé, esses vasos foram emitidos pela artéria proventricular dorsal e pelo ramo esquerdo da artéria celíaca através da artéria proventricular ventral, como foi observado nas aves deste trabalho.

SILVA et al. (2001) descreveram ramos da 
artéria celíaca para o coração na linhagem Avian Farms, o que não foi verificado neste estudo.

A divisão da artéria celíaca, em dois ramos, esquerdo e direito, foi descrita por BHADURI et al. (1957), SCHWARZE \& SCHRODER (1972), GETTY (1981) e BAUMEL (1993) em G. gallus; SILVA et al. (1996) na linhagem Hubbard; SILVA et al. (2001) na Avian Farms e MIRANDA et al. (2005) na Redbro Plumé, como foi observado em todos os espécimes desta pesquisa.

GETTY (1981) citou que, no G. gallus, o ramo esquerdo irrigou o fígado através da artéria hepática esquerda e o ramo direito emitiu a calibrosa artéria hepática direita, que vascularizou o fígado e a vesícula biliar, como foi observado nos avestruzes.

A irrigação do ventrículo na galinha doméstica ocorreu a partir do ramo esquerdo da artéria celíaca, denominado de artéria gástrica ventral por BAUMEL (1993), além de uma artéria para face esquerda do ventrículo (EDE, 1965), que foi nomeada por BHADURI et al. (1957) como artéria gástrica esquerda, com o que concordamos. MIRANDA et al. (2005) relataram que as artérias gástrica ventral e dorsal, esquerda e direita, na linhagem Redbro Plumé, irrigaram o ventrículo variando de seis a 12 colaterais. Essa variação ocorreu no presente trabalho de forma semelhante, porém de dois a 15 vasos.

O baço em G. gallus, segundo BHADURI et al. (1957), recebeu apenas uma delgada artéria esplênica, enquanto que NICKEL et al. (1977) citaram vários pequenos vasos, SCHWARZE \& SCHRODER (1972) relataram de duas a três artérias e GETTY (1981) descreveu a irrigação do baço como sendo efetuada pelas calibrosas artérias esplênicas cranial e caudal, não se detendo a numerálas. MIRANDA et al. (2005) verificaram que, na linhagem Redbro Plumé, o ramo direito irrigou o baço variando de dois a cinco, o que não aconteceu nos avestruzes, em que essas artérias foram emitidas através da celíaca em $100 \%$ dos casos, variando de um a dois; e em 3,3\% houve a contribuição do ramo esplênico através da artéria proventricular dorsal.

Em relação à artéria pancreaticoduodenal, GETTY (1981) na G.gallus; SILVA et al. (1996) na linhagem Hubbard; SILVA et al. (1997) na Ross; DRUMMOND et al. (2000) G. gallus domesticus; SILVA et al. (2001) na Avian Farms e MIRANDA et al. (2005) na Redbro Plumé, concordaram entre si que é uma continuação final do ramo direito da artéria celíaca e que emitiu vasos ao pâncreas e ao duodeno. Informamos ainda que, no avestruz, esses órgãos receberam irrigação diretamente do ramo direito, próximo à transição gastroduodenal, o pâncreas em 46,6\% das aves, variando de um a cinco, e o duodeno em $13,3 \%$, de um e dois.

$\mathrm{Na}$ galinha doméstica, as artérias ileocecais originaram-se a partir da pancreaticoduodenal, que foram citadas por EDE (1965) como ramos secundários; SCHWARZE \& SCHRODER (1972) relataram a emissão de uma artéria ileocecal após o envio das esplênicas, antes do surgimento da pancreaticoduodenal; GETTY (1981) fez referência às artérias ileocecais; com o que concordamos e acrescentamos que podem apresentar em número de um $(90 \%)$ a dois (10\%) e que sua contribuição para o íleo foi de um a 10 ramos, o ceco esquerdo, de dois a 10 , e o direito, de dois a nove.

\section{CONCLUSÕES}

A artéria celíaca originou-se da aorta descendente, sendo o seu primeiro ramo ventral e, dirigindo-se para o antímero direito em seu trajeto, enviou vasos para: esôfago, proventrículo, ventrículo, baço, fígado, vesícula biliar, pâncreas, duodeno, íleo, cecos esquerdo e direito.

\section{REFERÊNCIAS}

BAUMEL, J. J. Handbook of avian anatomy: Nomina anatômica avium. 2 ed. Massachusetts: Cambridge, 1993. p.407-421.

BHADURI, J. L.; BISWAS, B.; DAS, S. K. The arterial system of the domestic pigeon (Columba liva gmelin). Anatomischer Anzeiger, Deerfield Beach, v. 104, n. 14, p. 1-14, 1957.

CARBÓ, C. B. Producción del avestruz: aspectos claves. Madrid: Mundi Pressa, 2003. p. 7-30.80.

DRUMMOND, S. S.; CARDOSO, J. R.; SILVA, F. O. C.; SEVERINO, R. S.; MARTINS, A. K.; MOTA, F. C. D. Origem e distribuição da artéria celíaca em Gallus gallus domesticus. Veterinária Notícias, Uberlândia, v.6, n.1, p. 35-42, 2000.

EDE, D. A. Las vísceras. In: Anatomia de las aves. Zaragoza: Acribia, 1965. p.86-88.

GETTY, R. Sisson/ Grossman anatomia dos animais domésticos. 5. ed. Rio de Janeiro: Interamericana, 1981. 
v. 2. p. 1862-1869.

GIANNONI, M. L. Viabilidade da exploração de ratitas em São Paulo. São Paulo: Biológico, v. 1, n. 2, 1998. p. 91-96

MIRANDA, R. L.; SILVA, F. O. C.; LIMA, J. C. S.; CASTRO, J. R.; QUIRINO, R. C.; SEVERINO, R. S.; DRUMMOND, S. S. Origem e distribuição da artéria celíaca em aves (Gallus gallus) de corte da linhagem Redbro Plumé. Bioscience Journal, Uberlândia, v. 21, n. 3, p. 77-83, Spt./Dec. 2005.

MIRANDA, R. L.; SILVA, F. O. C.; SEVERINO, R. S.; DRUMMOND, S. S. SOLA, M. C.; MENDONÇA, E. P.; SILVA JÚNIOR, W. Origens e distribuições das artérias mesentéricas cranial e caudal em aves (Gallus gallus) da linhagem Bovans Goldline. Bioscience Journal, Uberlândia, v. 25, n. 1, p. 157-162, Jan./Feb. 2009.

NICKEL, R.; SCHUMER, A.; SEIFERLE, E. Circulatory system. In: Anatomy of the domestic birds. Berlim: Velag Paul Parey, 1977. p.85-107.

SCHWARZE, E.; SHRODER, L. Compêndio de anatomia veterinária. Zaragoza: Acribia, 1972. v.5, p. 64-74.

SICK, H. Ordem Rheiformes - emas: família Rheidae. In:
SICK, H. Ornitologia brasileira. Rio de Janeiro: Nova Fronteira, 1997. p. 168-171.

SILVA, F. O. C.; SEVERINO, R. S.; SANTOS, A. L. Q. ; DRUMMOND, S. S.; BOMBONATO, P. P.; SANTANA, M. I. S.; LOPES, D. Origem e distribuição da artéria celíaca em aves (Gallus gallus domesticus) da linhagem Avian Farms. Veterinária Notícias, Uberlândia, v. 7, n. 2, p. 27-36, 2001.

SILVA, F. O. C.; SEVERINO, R. S.; SANTOS, A. L. Q.; DRUMMOND, S. S.; BOMBONATO, P. P.; SANTANA, M. I. S.; LOPES, D.; MARÇAL, A. V. Origem e distribuição da artéria celíaca em aves (Gallus gallus domesticus linhagem Ross). Revista da Faculdade de Zootecnia, Veterinária e Agronomia, Uruguaiana, v.4, n.1, p.35-41, 1997.

SILVA, F. O. C.; SEVERINO, R. S.; SANTOS, A. Q.; DRUMMOND, S. S.; JÚNIOR, W, S.; BOMBONATO, P. P.; SANTANA, M. I. S.; CAMILO, V. Origem e distribuição da artéria celíaca em aves (matrizes de corte linhagem Hubbard). Veterinária Notícias, Uberlândia, v.2, n.1, p.17-24, 1996.

SISSON, S.; GROSSMAN, J. D. Anatomia de los animals domésticos. 4.ed. Barcelona: Salvat, 1975. p.920-923. 